Trauma Berufskrankh 2016 · [Suppl 1]: 18:S12-S17 DOI 10.1007/s10039-015-0097-2

Online publiziert: 10. November 2015

๑) Springer-Verlag Berlin Heidelberg 2015

CrossMark

Alexander von Glinski · Jan Geßmann · Matthias Königshausen •

Thomas A. Schildhauer. Dominik Seybold

Berufsgenossenschaftliches Universitätsklinikum, Bergmannsheil $\mathrm{GmbH}$, Bochum

\title{
Knorpeltrauma bei Schulterluxation
}

\section{Wie bestimmt es die Omarthroseentwicklung?}

Die Schulter ist das am häufigsten luxierte Gelenk des menschlichen Körpers. Besonders junge und sportliche Patienten erleiden eine traumatische Schulterluxation. Nach der adäquaten Erstversorgung stellt sich die Frage der Folgeschäden im Hinblick auf die Entstehung einer möglichen posttraumatischen Omarthrose. Der initiale Knorpelschaden sowie die adäquate Einschätzung dessen in Kombination mit den Reluxationsereignissen scheinen neben der optimalen chirurgischen Versorgung der Begleitläsionen ausschlaggebende Faktoren zu sein.

Das Schultergelenk ist durch die enorme Beweglichkeit und den damit verbundenen Bewegungsumfang charakterisiert. Erreicht wird dies durch das bestehende Größenverhältnis der Gelenkflächen des Humeruskopfes und der Fossa glenoidales sowie durch die Kombinationsbewegung thorakoskapulär. Somit handelt es sich um ein nicht formschlüssiges Gelenk. Eine ausreichende Stabilität wird durch ein komplexes Zusammenspiel aktiver und passiver Stabilisatoren in Form von Bändern, Muskeln und dem Kapselapparat erreicht. Aufgrund der anatomischen Besonderheiten ist das Glenohumeralgelenk das am häufigsten luxierte Gelenk des menschlichen Körpers: $45 \%$ der Gelenkluxationen betreffen das Schultergelenk. Die Inzidenz liegt laut Literatur zwischen 0,1 und $1,7 \%$ [15].

Im Weiteren unterscheidet man anhand der Luxationsrichtung zwischen vorderer und hinterer Luxation, wobei die vordere Luxation mit ca. $95 \%$ den größten Anteil ausmacht. Hinsichtlich der vorderen Luxation ist die weitaus häufigste die Luxatio subcoroidea. Die traumatische Schulterluxation ist von pathologischen Veränderungen im Bereich des KapselLabrum-Ligamentkomplexes der Schulter begleitet. Diese Begleitschäden unterliegen einem gewissen Muster abhängig vom Alter des Patienten. So kommt es bei jüngeren Patienten regelhaft zu einem Abriss im Bereich des vorderen Labrum glenoidale (Bankart-Läsion) in Kombination mit einer anterioinferioren plastischen Deformierung der Kapsel. Durch einen solchen Schaden der passiven Stabilisatoren des Schultergelenks steigt die Wahrscheinlichkeit einer Reluxation. Nach stattgehabter Erstluxation erfolgt nach sorgfältiger Dokumentation möglicher neurologischer Ausfälle insbesondere im Innervationsgebiet des $\mathrm{N}$. axillaris sowie radiologischen Ausschlusses einer möglichen knöchernen Verletzung die Reposition. Wir bevorzugen die modifizierte Technik nach Milch nach vorheriger intraartikulärer Analgesie mittels Xylocain $1 \%$. Nach postrepositioneller Röntgenkontrolle sowie Reevaluation des neurologischen Status wird je nach Verletzungsmuster, Aktivitätslevel und Alter des Patienten eine adäquate konservative oder operative Therapie gewählt. Nach traumatischer Schulterluxation kann es sowohl bei operativ stabilisierten als auch bei konservativ therapierten Patienten im Verlauf zur Schultergelenkarthrose kommen. Bereits 1983 prägten Samilson und Prieto [23] hierfür den Begriff der Luxationsarthropathie, die primär an- hand von nativradiologischen Kriterien klassifiziert wird.

Seit Längerem herrscht eine kontroverse Diskussion, ob die Entstehung einer Omarthrose nach Luxation bereits durch den primären Knorpelschaden vorbestimmt ist oder ob sie die Folge einer möglichen Instabilität darstellt. Einige Autoren sehen auch die operative Stabilisierung als Ursache der resultierenden Arthrose und geben eine Inzidenz von postoperativer Omarthrose zwischen 12 und $62 \%$ an $[1,7,18,22]$. Im Folgenden soll der Einfluss eines Knorpeltraumas im Rahmen der Erstluxation näher erörtert werden.

\section{Traumatische Erstluxation - sog. "first hit"}

Als „first hit“ wird der Knorpelschaden nach traumatischer Erstluxation bezeichnet. Hierbei treten verschiedene Verletzungsmuster auf. Grundsätzlich unterscheidet man zwischen

Tab. 1 Inzidenzen der Begleitpathologien nach traumatischer Schulterluxation. (Nach Boss et al. [2])

\begin{tabular}{|ll|}
\hline Verletzung & $\begin{array}{l}\text { Häufigkeit } \\
(\%)\end{array}$ \\
\hline Labrumläsion & 88 \\
\hline Hill-Sachs-Läsion & 54 \\
\hline SLAP-Läsion & 11 \\
\hline $\begin{array}{l}\text { Rupturen der } \\
\text { Rotatorenmanschette }\end{array}$ & 22 \\
\hline $\begin{array}{l}\text { Frakturen des Tuberculum } \\
\text { majus }\end{array}$ & 4 \\
\hline SLAP ${ }_{\text {,Superior labrum anterior to inferior“. }}$ \\
\hline
\end{tabular}




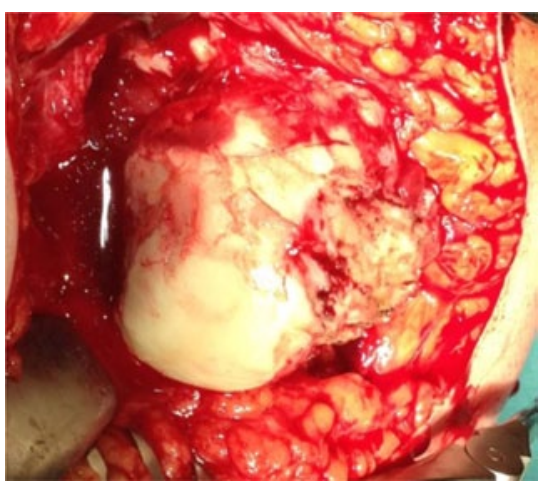

Abb. 1 \& Hill-Sachs-Läsion

isolierten Knorpelabscherverletzungen z. B. bei einer vorderen Luxation im anteroinferioren Bereich. Weiterhin entstehen verschiedene Labrum-Ligamentläsionen mit Beteiligung des Knochens bzw. des Knorpels.

Im Rahmen einer chondralen Beteiligung bei Labrumläsion spricht man von einer "glenolabral articular disruption" (GLAD). Kommt es zu einer ossären Beteiligung, die superior bis in den Bizepssehnenanker einstrahlt, so spricht man von einer SLAP („superior labrum anterior to inferior")-Läsion. Kommt es zur vollständigen Trennung des anteroinferioren Kapsel-LabrumKomplexes spricht man von einer Bankart-Läsion bzw. bei zusätzlichem Ausriss von ossären Anteilen von einer knöchernen Bankart-Läsion. Da die Reißfestigkeit der Gelenkkapsel mitunter die Festigkeit des ossären Glenoids übersteigt, kommt es teilweise auch zu knöchernen Bankart-Läsion, also dem Labrumabriss mit knöcherner Beteiligung. Kommt es zusätzlich zu einer subperiostalen Ablösung des inferioren glenohumeralen Ligaments, handelt es sich um eine sog. Perthes-Läsion. Letztlich muss nach traumatischer Schulterluxation auch humeralseitig nach einer Ablösung des dort anliegenden Kapsel-Band-Apparates gesucht werden, was als HAGL (,humeral avulsion of glenohumeral ligaments")Läsion bezeichnet wird.

Auch im Bereich des Humeruskopfes kommt es nach Luxation durch Druckwirkung des Glenoidrandes zu KnorpelKnochen-Schäden. Bei der häufigeren vorderen Luxation entsteht so eine charakteristische Impression im Bereich des dorsokranialen Humeruskopfes,

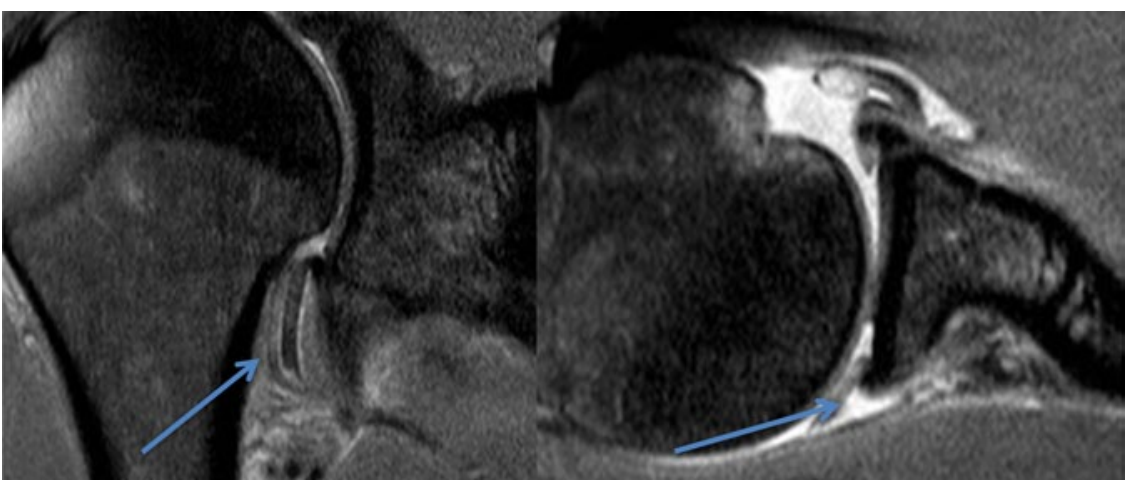

Abb. 2 ム Umgeschlagene Gelenkknorpelanteile (Pfeile)

die als Hill-Sachs-Läsion ( $\bullet$ Abb. 1) bezeichnet wird. Die Inzidenz liegt bei Erstluxation bei $67 \%$ [25]. Analog dazu bezeichnet man nach stattgehabter hinterer Luxation den ventralen Schaden als „reversed“ Hill-Sachs-Läsion oder Malgaigne-Läsion.

Das Auftreten möglicher Begleitverletzungen lässt sich $u$. a durch die Arbeit von Boss et al. [2] abschätzen (• Tab. 1).

Nach einer Erstluxation gilt es, neben der Labrum-Ligamentläsion den entstandenen Knorpelschaden zu evaluieren. Das MRT bietet hier mit hochauflösenden Knorpelsequenzen eine gute Ortsauflösung und Knorpelbeurteilung. Zur möglichen Objektivierung anhand der MRT-Aufnahmen dient das Hyaline Cartilage Lesion Classification System der International Cartilage Repair Society (ICRS). Hier wird zum einen die Tiefe der Läsion, zum anderen der Ort der Läsion berücksichtigt. Makroskopisch unauffälliger Knorpel wird als IRCS 0 bewertet. Im Fall einer intakten Oberfläche mit jedoch makroskopisch sichtbarer Eindellung spricht man von IRCS $1 \mathrm{a}$, im Fall von Fissuren in der Oberfläche von 1b. Sollte ein Defekt weniger als $50 \%$ der Dicke des Knorpels betreffen, spricht man von IRCS 2, bei einer Tiefe von mehr als $50 \%$ von IRCS 3. Ist der subchondrale Knochen beteiligt, so spricht man von IRCS 4 ([3]; • Abb. 2).

Eine genaue Beurteilung und v. a. Einteilung des Knorpelschadens ist jedoch nur arthroskopisch möglich. Die bekannteste Klassifikation von Knorpelschäden auf Grundlage der arthroskopischen Befunde stammt von Outerbridge aus dem Jahr 1961. Es werden 4 Grade des Schadens unterschieden. Grad 1 beschreibt weichen ggf. geschwollenen Knorpel, Grad 2 einen Schaden der Oberfläche mit Fissuren, die sich nicht bis in den subchondralen Knochen erstrecken und einen Diameter von $1,5 \mathrm{~cm}$ nicht überschreiten. Geschieht dies, spricht man von einem Grad 3. Sollte der subchondrale Knochen exponiert sein handelt es sich um einen Schaden von Grad 4. Es gilt als problematisch, dass bei Grad 3 und 4 nicht nach der Tiefe des Knorpelschadens differenziert wird. Zudem wird die Lokalisation des Knorpelschadens nicht berücksichtigt.

\section{Auswirkung der Lokalisation des Knorpelschadens}

Entscheidend für die Auswirkung eines möglichen Knorpelschadens und der möglicherweise daraus resultierenden Arthrose ist neben der Tiefe und der betroffenen Fläche auch die Lokalisation. Letztere spielt eine entscheidende Rolle hinsichtlich der Verfahrensweise im Rahmen einer möglichen Stabilisierungsoperation. Es konnte gezeigt werden, dass durch einen Knochenverlust besonders im Bereich des anterior inferioren Quadranten des Glenoids die Kontaktfläche zwischen Glenoid und Humeruskopf drastisch reduziert wird [13]. Im Fall eines Defekts von 30\% des Diameters des inferioren Glenoids kommt es somit zu einer durchschnittlichen Erhöhung des Anpressdruckes von $100 \%$ im Bereich des gesamten Glenoids bzw. von $300 \%$ im Bereich des inferioren Anteils. Durch die vermehrte Umverteilung des Druckes auf das ggf. refixierte Labrum kommt es häufiger zu revisionsbedürftigen operativen Ergebnissen. 
Zudem trägt der inferiore bauchige Teil des Glenoids („pear“) maßgeblich zur Stabilität bei. Im Fall einer großen knöchernen Bankart-Läsion oder aber einer Impression des Knorpels im unteren Bereich des Glenoids kommt es zur Umkehr der Form, und es entsteht ein sog. „inverted pear glenoid“. Ab einem Substanzverlust von $>25 \%$ im Bereich des unteren Diameters des Glenoids besteht in der Literatur weitestgehend Einigkeit, dass eine operative Intervention zur Rekonstruktion der Gelenkfläche nötig ist, um eine Stabilität des Gelenks zu gewährleisten [17].

Betrachtet man die knöchernen Substanzverluste im Bereich des Humeruskopfes isoliert, gibt es keine eindeutige Empfehlung, ab welcher Defektgröße eine Instabilität entsteht. In verschiedenen Studien konnte jedoch gezeigt werden, dass ab einer Beteiligung von $20-40 \%$ der humeralen Gelenkfläche ein Stabilitätsverlust mit resultierendem Luxationsrisiko aufritt und somit eine operative Augmentation erforderlich wird $[8,9]$.

\section{„Bipolar bone loss"}

Eine alleinige Betrachtung von Glenoidläsion und Hill-Sachs-Läsion ist nicht sinnvoll, da ein kleiner Glenoiddefekt in Kombination mit einer großen Hill-Sachs-Läsion schon stabilitätsbeeinflussend sein kann und umgekehrt ein größerer Glenoiddefekt bei kleiner Hill-Sachs-Läsion stabil erscheint (- Abb. 3).

Eine Möglichkeit, das Zusammenspiel der Läsionen zu beurteilen, bietet das Konzept von Burkhart und DeBeer [4]. Sie unterschieden zwischen Engagingund Non-engaging-Läsionen im Bereich des Humeruskopfes. Hierzu wird die Schulter im Rahmen einer Arthroskopie dynamisch untersucht (in $90^{\circ}$ Abduktion sowie in maximaler Außenrotation in einem Bereich von $0-135^{\circ}$ ). Sollte die Hill-Sachs-Läsion sich mit dem vorderen Rand des Glenoids im Rahmen der Bewegung überschneiden, gilt die Läsion als ,engaging“. Patienten mit diesem Verletzungsmuster wiesen nach isoliertem Bankart-Repair eine rezidivierende Instabilität von $67 \%$ auf im Vergleich zu $4 \%$

Trauma Berufskrankh 2016 • [Suppl 1]: 18:S12-S17 DOI 10.1007/s10039-015-0097-2

(c) Springer-Verlag Berlin Heidelberg 2015

A. von Glinski · J. Geßmann · M. Königshausen · T.A. Schildhauer · D. Seybold

\section{Knorpeltrauma bei Schulterluxation. Wie bestimmt es die Omarthroseentwicklung?}

\section{Zusammenfassung}

Aufgrund der anatomischen Besonderheiten sind Luxationen des Glenohumeralgelenks häufig. Verschiedene Prädispositionsfaktoren einer möglichen Dislokationsarthropathie werden in der Literatur diskutiert. Grundsätzlich entsteht der größte Knorpelschaden im Rahmen des sog.,first hit", der traumatischen Erstluxation. Das Alter zum Zeitpunkt der Erstluxation und auch zum Zeitpunkt einer operativen Stabilisierung scheint einen Einfluss auf die Entstehung einer möglichen Arthropathie zu haben. Nicht die Dauer einer möglichen Instabilität vor operativer Stabilisierung, sondern vielmehr die rezidivierenden Luxationen haben einen weiteren Einfluss auf den Gelenkverschleiß. Eine operative Stabilisierung stellt, sofern technisch gut ausgeführt, kein er- höhtes Arthroserisiko dar, ist jedoch nicht in der Lage, eine Entwicklung der Arthropathie zu verhindern. Es gelingt jedoch, durch diese eine Rezidivluxation zu vermeiden und somit den Schweregrad einer Dislokationsarthropathie zu verringern. Bei der Planung einer möglichen Stabilisierung ist es essenziell, den Knorpelschaden im Bereich des Humeruskopfes sowie im Bereich des Glenoids zu evaluieren („,bipolar bone loss") und das dynamische Verhältnis dieser Läsionen zueinander zu berücksichtigen.

\section{Schlüsselwörter}

Schulterdislokation .

Dislokationsarthropathie · Bankart-Läsion .

Arthropathie · Glenohumeralgelenk

\section{Chondral damage after traumatic dislocation of the shoulder. How does it determine the development of arthrosis?}

\begin{abstract}
Dislocation of the shoulder joint is a frequent occurrence due to the anatomical features. Various predisposing factors that determine the prevalence of dislocation athropathy have been discussed in the literature. Principally, the greatest chondral damage occurs during the initial traumatic shoulder dislocation, the so-called first hit. The age of the person at the time of the first hit and age at the time of surgery in particular seem to influence the development of dislocation arthropathy. The duration of shoulder instability before surgery does not seem to affect the incidence of athropathy. An operation, if technically well performed, does not increase the
\end{abstract}

risk of arthropathy and furthermore, it prevents redislocation and therefore decreases the risk of dislocation athropathy. What is important is how often redislocation occurs. When planning a possible surgical stabilization of the shoulder it is crucial to assess the degree of damage in the region of the humeral head and the glenoid cavity (bipolar bone loss) and the dynamic interrelationship of these lesions.

\section{Keywords}

Shoulder dislocation · Dislocation athropathy · Arthropathy · Glenohumeral joint $\cdot$ Bankart lesion bei Patienten ohne den oben genannten Defekt [4]. Grundsätzlich ist es jedoch problematisch, das Artikulationsverhältnis von Glenoid und Humeruskopf nach stattgehabter Luxation im Rahmen einer Arthroskopie zu ermitteln, da hierbei oft ein gedehnter Kapsel-Band-Apparat vorliegt und somit die Kontaktfläche nicht identisch ist mit der bei intaktem KapselBand-Apparat. Im Umkehrschluss birgt die Kontrolle nach durchgeführter Bankart-Repair die Gefahr einer möglichen Dehnung/eines Schadens an dem frisch operierten kapsuloligamentären Komplex.

Komplementär zu dem Konzept von Burkhardt et al. untersuchten Itoi et al. [29] zunächst die anatomische Lagebeziehung zwischen Humeruskopf und Glenoid in verschiedenen Positionen der Schulter mit intaktem Kapsel-BandApparat. Im Weiteren untersuchten sie mithilfe von 3-dimensionalen CTAufnahmen die Lagebeziehung der bipolaren Knochendefekte in maximaler Abduktion und Außenrotation. Im 


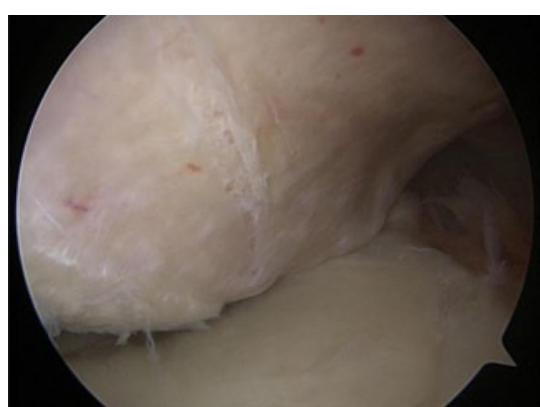

Abb. $3 \Delta$ Kombinierte Hill-Sachs-Läsion und Glenoiddefekt

Rahmen der oben genannten Bewegung wandert die Kontaktfläche zwischen Humeruskopf und Glenoid auf dem Humeruskopf von inferiomedial nach superolateral. Diese dynamische Kontaktfläche wird als "glenoid track“ definiert. Sollte eine Hill-Sachs-Läsion die Fläche des "glenoid track“ überschreiten, spricht man von einer Offtrack-Läsion, ansonsten von einer Ontrack-Läsion. Um den „glenoid track“ zu bestimmen, muss zunächst der Defekt im Bereich des Glenoids ausgemessen werden und von $83 \%$ der ursprünglichen Glenoidbreite subtrahiert werden. Die Messung kann entweder arthroskopisch oder mittels 3-dimensionaler CT erfolgen. Nun müssen die Breite der Hill-Sachs-Läsion sowie die Weite der Knochenbrücke zwischen dem Rand der Hill-Sachs-Läsion und dem Ansatz der Rotatorenmanschette gemessen und addiert werden. Sollte dieser Wert größer als der errechnete "glenoid track" sein, so ist die Läsion als Off-track-Läsion anzusehen [10]. Itoi et al. nutzen diese Information in Kombination mit dem prozentual betroffenen Glenoiddefekt, um eine Entscheidung für oder gegen eine operative Sanierung in Form eines Bankart-Repairs bzw. eines knöchernen Verfahrens zu treffen. Somit ist nach stattgehabtem Schaden im Bereich des Knorpels eine Aussage über resultierende Instabilitäten möglich. Doch wie ist der Zusammenhang zwischen einer bestehenden Instabilität und einer resultierenden Omarthrose, und gibt es möglicherweise Risikofaktoren? Und welchen Einfluss hat der initiale Knorpelschaden hierbei?

Es gilt als gesichert, dass bereits ein 1-maliges Luxationsereignis des Schultergelenks die Wahrscheinlichkeit einer

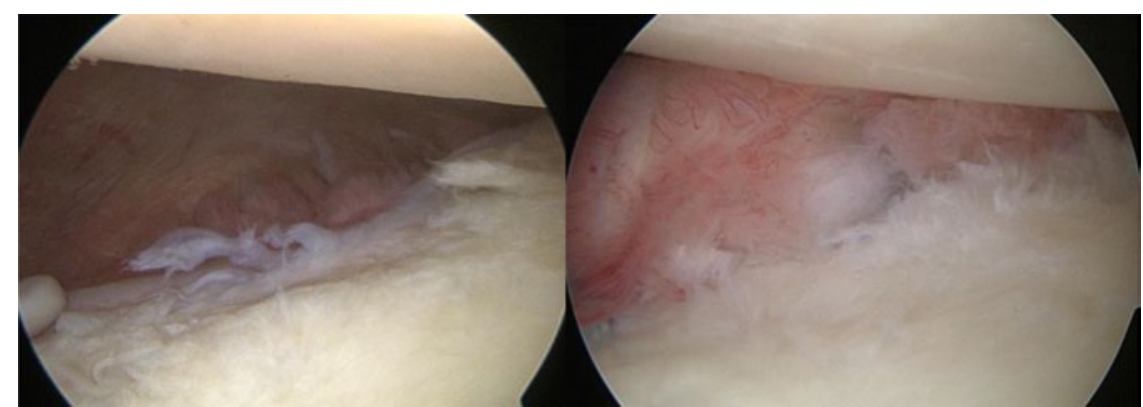

Abb. $4 \Delta$ GLAD ("glenolabral articular disruption“)-Läsion mit anschließender Refixation und gutem Labrum-Glenoid-Schluss

späteren Omarthrose erhöht. So konnten Samilson et al. [23] zeigen, dass bereits nach 1-maliger Luxation und 10-JahresFollow-up eine erhöhte Arthroserate bei instabilen Schultergelenken im Vergleich zu stabilen Gelenken von $20 \%$ vorliegt ( $11 \%$ mild, $9 \%$ moderat bzw. schwer). Der Langzeitverlauf nach Schulterluxation in Bezug auf das Auftreten möglicher Omarthrosen konnte in einer Studie von Hovelius et al. [16] erörtert werden. Klassifiziert anhand der radiologischen Einteilung von Samilson et al., wurde bei $26 \%$ der Patienten nach 25 Jahren eine moderate bis schwere Dislokationsarthropathie nachgewiesen. Betrachtet man zusätzlich die Patienten mit einer milden Arthropathie, so sind $56 \%$ der Patienten betroffen. Als mögliche Risikofaktoren wird die Erstluxation mit einem Alter $<25$ Jahren genannt. Im Weiteren zählen Alkoholiker zu den Risikopatienten. Das unter Alkoholeinfluss vermeidlich unkontrollierte Trauma soll - ähnlich wie ein Hochrasanztrauma bei Athleten - einen größeren Knorpelschaden im Rahmen des „first hit“ produzieren [16]. Unklar bleibt in genannten Studien jedoch die Inzidenz einer zweiten Luxation bei bestehender Instabilität.

\section{"Second hit"}

Betrachtet man mögliche Einflussfaktoren einer Omarthroseentwicklung nach Erstluxation, ist die Auswirkung eines „second hit“ zu berücksichtigen. Hierbei kann es sich um einen erneuten Knorpelschaden im Rahmen einer Reluxation handeln oder aber um einen möglichen Knorpelschaden durch das operative Vorgehen. Beides kann die Ent- wicklung einer Dislokationsarthropathie beeinflussen.

Das alleinige Vorliegen einer Instabilität ohne weitere Dislokationen scheint kein ausschlaggebender Risikofaktor einer Dislokationsarthropathie zu sein. Cameron et al. [6] untersuchten 422 Patienten mit Schulterinstabilität und unterteilten diese in akute ( $<3$ Monate) und chronische ( $>3$ Monate) Instabilität. Sie fanden keinen signifikanten Unterschied hinsichtlich der Arthroserate.

In einer Langzeitstudie von Privitera et al. [20] wurde nach 13 Jahren eine mittelgradige bis schwere Arthropathie bei $40 \%$ der Patienten nach arthroskopischer Bankart-Operation beschrieben. Auch Plath et al. [19] konnten bei 100 Patienten mit arthroskopisch erfolgtem BankartRepair und einem durchschnittlichen Follow-up von 13 Jahren bei $69 \%$ Zeichen einer Arthrose nachweisen. Vergleicht man dies mit der von Hovelius et al. [15] beschriebenen Arthroserate von $60 \%$ bei nichtoperativem Vorgehen, so scheint eine operative Stabilisierung die Entwicklung einer Dislokationsarthropathie nicht zu verhindern. Problematisch bleibt die Tatsache, dass mögliche Reluxationen während der bestehenden Instabilität nicht näher erläutert werden und somit der Einfluss eines erneuten Knorpelschadens durch Reluxation nicht berücksichtigt wird. In der Literatur wird eine deutliche Korrelation der Reluxationen vor operativer Stabilisierung und der resultierenden Dislokationsarthropathie beschrieben [5, 11, 19]. Somit ist der Benefit einer operativen Stabilisierung hinsichtlich einer möglichen Arthropathie v. a. in der Vermeidung möglicher Rezidive zu sehen. 
Insbesondere im jugendlichen Alter und bei Ausüben einer Kontaktsportart besteht ein hohes Rezidivrisiko. Somit liegt es nahe, v. a. junge (bei Hovelius et al. unter 25 Jahre) und sportlich aktive Menschen primär stabilisierend $\mathrm{zu}$ operieren. Ziel hierbei ist es, den „second hit" in Form einer erneuten Luxation und den so entstehenden zusätzlichen Knorpelschaden $\mathrm{zu}$ verhindern. So konnte die Rezidivdislokation bei Hochrisikopatienten (Kadetten einer Militärakademie der USA) durch primär stabilisierende Operation im Vergleich zur konservativen Therapie signifikant reduziert werden [27].

Wie eingangs erwähnt, ist ein „second hit" auch im Rahmen des operativen Vorgehens möglich. Es steht zur Diskussion, welchen Einfluss die Stabilisierungsoperation auf die Entwicklung einer Omarthrose nimmt. Bereits bekannt ist, dass durch eine operativ bedingte Einschränkung bzw. den Verlust der Außenrotation - beispielsweise durch eine mediolaterale Kapselverkürzung - ein erhöhtes Risiko einer Sekundärarthrose entsteht. Bei korrekter Operationsindikation und Technik zeigt sich in der Literatur jedoch keine erhöhte Inzidenz einer Arthrose im Vergleich zu der konservativen Therapie [16]. Voraussetzung ist hier eine korrekte Rekonstruktion der anatomischen Verhältnisse. So konnte in der Studie von Hovelius et al. bei 62 stabilisierten Schultern keine erhöhte Arthropathierate im Vergleich zu den konservativ therapierten Schultern gefunden werden. Ausschlaggebende Kriterien für ein gutes operatives Ergebnis sind neben der Knotentechnik des Operateurs und der Positionierung der Knoten auch die Anzahl und die Art der Anker. Zunächst betrachten wir die Rolle der Ankeranzahl. Hierbei bleibt zu berücksichtigen, dass der Grad des Labrumschadens einen weiteren bekannten Risikofaktor für die langfristige Entwicklung einer Arthropathie darstellt [11]. Die Verwendung mehrerer Anker impliziert einen höhergradigen Schaden im Bereich des Labrums und somit ggf. ein höheres Risiko der späteren Entwicklung einer höhergradigen Arthropathie. Bezüglich der postoperativen Stabilität im Zusammenhang mit der Ankeranzahl postulieren Van der Linde et al. [26] eine reduzierte Reluxationsrate durch die Verwendung von 3 statt 2 Ankern. Problematisch scheint die von einigen Autoren beschriebene Korrelation zwischen der Anzahl der verwendeten Anker und möglichen Folgeschäden zu sein. Nach Verwendung von metallischen Ankern kann es durch eine Fehllage zu degenerativen Veränderungen aufgrund mechanischer Komplikationen kommen [21]. Es konnte Ähnliches auch bei der Verwendung von resorbierbaren Ankern festgestellt werden [24]. Ein möglicher Vorteil der Verwendung mehrerer Anker liegt möglicherweise in besserer Stabilität. Eine aktuelle Studie aus dem Jahr 2014 von Witney-Lagen et al. [28] konnte jedoch keinen signifikanten Unterschied hinsichtlich der Luxationsraten bei der Verwendung von 1 bzw. 2 und 3 Ankern finden. Als entscheidender Faktor für die Stabilität wird der LabrumGlenoid-Schluss durch die superomediale Verlagerung des gerissenen Labrums bzw. der Gelenkkapsel im Sinne einer Raffung und Aufwulstung der Kapsel am Glenoidrand postuliert (• Abb. 4).

Neben der optimalen Operationstechnik wurden auch die möglichen Zugangsmorbiditäten verglichen. Die Gruppe um Frank et al. [12] verglich den Zugang in Beachchair-Position mit dem in Seitenlage. Zwar ließen sich geringere Rezidivraten für Stabilisationen in Seitenlage feststellen, jedoch auch vermehrte lagerungsbedingte Komplikationen in Form von Lagerungsschäden (Traktionsneuropathien). Die Autoren sehen somit leichte Vorteile für die Seitlage.

\section{Fazit für die Praxis}

Eine Schultergelenkarthrose ist eine
mögliche Spätfolge einer Schulter-
gelenkluxation, wobei neben dem
initialen Trauma das fortgeschrittene
Alter zum Zeitpunkt der Erstluxation
einen Risikofaktor darstellt.
Die operative Stabilisierung des
Schultergelenks bei bestehender In-
stabilität scheint die Entstehung einer
Dislokationsarthropathie in Langzeit-
untersuchungen nicht zu verhindern.

Jedoch ist insbesondere bei aktiven Patienten das bestehende Risiko einer Reluxation zu berücksichtigen, das als sog. „,second hit" das Risiko einer posttraumatischen Omarthrose erhöht.

- Bei der Wahl des Stabilisierungsverfahrens gilt es, neben der Evaluierung der Schäden am Labrum-Ligamentkomplex der Schulter auch den Knochen-/Knorpelschaden sowohl im Bereich des Glenoids als auch im Bereich des Humeruskopfes gemeinsam und unter dynamischen Bedingungen zu evaluieren, um somit das geeignete Therapieverfahren zu finden.

\section{Korrespondenzadresse}

\section{Seybold}

Berufsgenossenschaftliches

Universitätsklinikum, Bergmannsheil $\mathrm{GmbH}$

Bürkle-de-la-Camp-Platz 1, 44789 Bochum

dominik.seybold@bergmannsheil.de

\section{Einhaltung ethischer Richtlinien}

Interessenkonflikt. A. von Glinski, J. Geßmann, M. Königshausen, T.A. Schildhauer und D. Seybold geben an, dass kein Interessenkonflikt besteht.

Dieser Beitrag beinhaltet keine Studien an Menschen oder Tieren.

The supplement containing this article is not sponsored by industry.

\section{Literatur}

1. Allain J, Goutallier D, Glorion C (1998) Long-term results of the Latarjet procedure for the treatment of anterior instability of the shoulder. J Bone Joint Surg Am 80:841-852

2. Boss A, Pellegrini L, Hintermann B (2000) [Prognostically relevant factors in treatment of the post-traumatic unstable shoulder joint]. Unfallchirurg 103:289-294

3. Brittberg M, Winalski CS (2003) Evaluation of cartilage injuries and repair. J Bone Joint Surg Am 85-A(Suppl 2):58-69

4. Burkhart SS, De Beer JF (2000) Traumatic glenohumeral bone defects and their relationship to failure of arthroscopic Bankart repairs: significance of the inverted-pear glenoid and the humeral engaging Hill-Sachs lesion. Arthroscopy 16:677-694

5. Buscayret F, Edwards TB, Szabo l et al (2004) Glenohumeral arthrosis in anterior instability before and after surgical treatment: incidence and contributing factors. Am J Sports Med 32:11651172 
6. Cameron ML, Kocher MS, Briggs KK et al (2003) The prevalence of glenohumeral osteoarthrosis in unstable shoulders. Am J Sports Med 31:53-55

7. Chapnikoff D, Besson A, Chantelot $C$ et al (2000) [Bankart procedure: clinical and radiological longterm outcome]. Rev Chir Orthop Reparatrice Appar Mot 86:558-565

8. Chen AL, Hunt SA, Hawkins RJ et al (2005) Management of bone loss associated with recurrent anterior glenohumeral instability. Am J Sports Med 33:912-925

9. Cho SH, Cho NS, Rhee YG (2011) Preoperative analysis of the Hill-Sachs lesion in anterior shoulder instability: how to predict engagement of the lesion. Am J Sports Med 39:2389-2395

10. Di Giacomo G, Itoi E, Burkhart SS (2014) Evolving concept of bipolar bone loss and the Hill-Sachs lesion: from „engaging/non-engaging "lesion to "on-track/off-track" lesion. Arthroscopy 30:90-98

11. Franceschi F, Papalia R, Del Buono A et al (2011) Glenohumeral osteoarthritis after arthroscopic Bankart repair for anterior instability. Am J Sports Med 39:1653-1659

12. Frank RM, Saccomanno MF, Mcdonald LS et al (2014) Outcomes of arthroscopic anterior shoulder instability in the beach chair versus lateral decubitus position: a systematic review and metaregression analysis. Arthroscopy 30:1349-1365

13. Greis PE, Scuderi MG, Mohr A et al (2002) Glenohumeral articular contact areas and pressures following labral and osseous injury to the anteroinferior quadrant of the glenoid. J Shoulder Elbow Surg 11:442-451

14. Habermeyer P, Gleyze P, Rickert M (1999) Evolution of lesions of the labrum-ligament complex in posttraumatic anterior shoulder instability: a prospective study. J Shoulder Elbow Surg 8:66-74

15. Hovelius $L$ (1982) Incidence of shoulder dislocation in Sweden. Clin Orthop Relat Res 66:127-131

16. Hovelius L, Saeboe M (2009) Neer award 2008: arthropathy after primary anterior shoulder dislocation-223 shoulders prospectively followed up for twenty-five years. J Shoulder Elbow Surg 18:339-347

17. Lo IK, Parten PM, Burkhart SS (2004) The inverted pear glenoid: an indicator of significant glenoid bone loss. Arthroscopy 20:169-174

18. O'neill DB (1999) Arthroscopic Bankart repair of anterior detachments of the glenoid labrum. A prospective study. J Bone Joint Surg Am 81:13571366

19. Plath JE, Aboalata M, Seppel G et al (2015) Prevalence of and risk factors for dislocation arthropathy: radiological long-term outcome of arthroscopic Bankart repair in 100 shoulders at an average 13-year follow-up. Am J Sports Med 43:1084-1090

20. Privitera DM, Bisson LJ, Marzo JM (2012) Minimum 10-year follow-up of arthroscopic intra-articular Bankart repair using bioabsorbable tacks. Am J Sports Med 40:100-107

21. Rhee YG, Lee DH, Chun IH et al (2004) Glenohumeral arthropathy after arthroscopic anterior shoulder stabilization. Arthroscopy 20:402-406

22. Rosenberg BN, Richmond JC, Levine WN (1995) Long-term followup of Bankart reconstruction. Incidence of late degenerative glenohumeral arthrosis. Am J Sports Med 23:538-544

23. Samilson RL, Prieto V (1983) Dislocation arthropathy of the shoulder. J Bone Joint Surg Am 65:456-460
24. Solomon DJ, Navaie M, Stedje-Larsen ET et al (2009) Glenohumeral chondrolysis after arthroscopy: a systematic review of potential contributors and causal pathways. Arthroscopy 25:1329-1342

25. Spatschil A, Landsiedl F, Anderl W et al (2006) Posttraumatic anterior-inferior instability of the shoulder: arthroscopic findings and clinical correlations. Arch Orthop Trauma Surg 126:217222

26. Van Der Linde JA, Van Kampen DA, Terwee CB et al (2011) Long-term results after arthroscopic shoulder stabilization using suture anchors: an 8to 10-year follow-up. Am J Sports Med 39:23962403

27. Wheeler JH, Ryan JB, Arciero RA et al (1989) Arthroscopic versus nonoperative treatment of acute shoulder dislocations in young athletes. Arthroscopy 5:213-217

28. Witney-Lagen C, Perera N, Rubin S et al (2014) Fewer anchors achieves successful arthroscopic shoulder stabilization surgery: 114 patients with 4 years of follow-up. J Shoulder Elbow Surg 23:382387

29. Yamamoto N, Itoi E, Abe H et al (2007) Contact between the glenoid and the humeral head in abduction, external rotation, and horizontal extension: a new concept of glenoid track. J Shoulder Elbow Surg 16:649-656 